\title{
Mitochondrial and nuclear genes as the cause of complex I deficiency
}

\author{
A.R. Rezaee ${ }^{1}$, A. Azadi', ${ }^{2,3}$, M. Houshmand ${ }^{4}$, F. Mahmoodi ${ }^{2}$, Z. Purpak ${ }^{3}$, \\ S. Safaei ${ }^{3}$, P. Karimi ${ }^{3}$, M. Ghabaee ${ }^{5}$ and M.A. Sahraian ${ }^{6}$ \\ ${ }^{1}$ Animal Science Department, College of Agriculture, \\ Ferdowsi University of Mashhad, Mashhad, Iran \\ ${ }^{2}$ Department of Biology, Azarbaijan University of Tarbiat Moallem, \\ Tabriz, Iran \\ ${ }^{3}$ Immunology, Asthma and Allergy Research Institute, \\ Tehran University of Medical Sciences, Tehran, Iran \\ ${ }^{4}$ National Institute for Genetic Engineering and Biotechnology, Tehran, Iran \\ ${ }^{5}$ Department of Neurology, Iranian Center of Neurological Research, \\ Tehran University of Medical Sciences, Tehran, Iran \\ ${ }^{6}$ Department of Neurology, Sina Hospital, \\ Iranian Center of Neurological Research, \\ Tehran University of Medical Sciences, Tehran, Iran \\ Corresponding author: M. Houshmand \\ E-mail: massoudh@nigeb.ac.ir
}

Genet. Mol. Res. 12 (3): 3551-3554 (2013)

Received March 30, 2013

Accepted August 9, 2013

Published September 12, 2013

DOI http://dx.doi.org/10.4238/2013.September.12.1

\begin{abstract}
Multiple sclerosis (MS) is an immunological inflammatory disease of the central nervous system. The pathogenesis of MS is incompletely understood, but various studies have suggested that mitochondrial dysfunction is associated with the disease. Mitochondria are among the main cellular sources of reactive oxygen and nitrogen species, and they play a pivotal role in many neuro-pathological conditions. The mitochondrial nuclear subunit of complex I gene in mitochondria may play a role in MS, and understanding this role may provide rationale for novel approaches to treatment of the disease and the development of novel therapies. We designed a molecular study to
\end{abstract}


demonstrate biochemical defects in complex I activity and found some novel nucleotide substitutions in mitochondrial DNA that might be involved in the pathogenesis of MS. The mitochondrial complex subunit I sequence was amplified and sequenced in MS patients. Although no reported pathogenic mutations were found in these patients, other studies have clearly indicated that the mitochondrial nuclear complex subunit I gene plays a significant role in MS pathogenesis.

Key words: Multiple sclerosis; Mitochondrial nuclear gene complex I; Pathogenic mutation

\section{INTRODUCTION}

Multiple sclerosis (MS) is a chronic disease of the central nervous system (Ghabaee et al., 2009) characterized by demyelization and devastation of axons (Mao and Reddy, 2010). The mitochondrion is an organelle that is present in all human cells (DiMauro and Schon, 2003; Kumleh et al., 2006). Mitochondria have an inner and an outer membrane. The respiratory chain, located in the inner membrane, is composed of 5 enzyme complexes (DiMauro and Schon, 2001; Zeviani and Di Donato, 2004). The respiratory chain is controlled by 2 separate genetic systems in the genomic and mitochondrial DNA (mtDNA). Respiratory complexes I, II, III, and IV contain both nuclear and mtDNA-encoded polypeptides. mtDNA is a small, closed circular and double-stranded molecule containing 37 genes, of which 24 are needed for translation (including 2 ribosomal RNAs and 22 transfer RNAs) and 13 encode subunits of the respiratory chain (DiMauro and Schon, 2001). Complex I, or nicotinamide adenine dinucleotide (NADH)-ubiquinone oxidoreductase, is composed of at least 46 subunits and its defects are the most frequent deficiencies of the respiratory chain (Smeitink et al., 2001). Lebers hereditary optic neuropathy is the mitochondrial disease in which mutations in mtDNA NADH genes were first detected (Fauser et al., 2002). Biochemical studies have established that catalytic activity of complex I in MS patients is significantly decreased compared with that in control subjects (Kumleh et al., 2006). Therefore, mutation of mitochondrial complex I subunit genes in MS patients may be an expected occurrence.

\section{MATERIAL AND METHODS}

We investigated 14 clinically diagnosed MS patients and 100 healthy normal controls. All of the patients were referred from the Iranian Center of Neurological Research and confirmed according to the McDonald criteria. The initial presenting symptom in the MS patients was optic neuritis. The Extended Disability Status Scale values of the patients ranged from 2.4 to 8 . Patients ranged in age from 24 to 37 years, and the female/male ratio was 1.8:1.

Consent forms were signed by parents and probands, and then peripheral blood samples were obtained, DNA was purified using a DNA extraction kit (Diatom, Gene Fanavaran, Tehran, Iran), and mtDNA was amplified using the primers listed in Table 1. The polymerase chain reaction (PCR) solution contained $0.4 \mu \mathrm{L}$ of each primer, $0.2 \mu \mathrm{L}$ Super Taq polymerase (Invitrogen, USA), $0.8 \mu \mathrm{L} \mathrm{MgCl}_{2}, 0.4 \mu \mathrm{L}$ master mix deoxyribonucleotide triphosphate, 10 $\mathrm{mM}$ of each deoxyribonucleotide triphosphate, and $2.5 \mu \mathrm{L}$ 10X PCR buffer in a final volume of $25 \mu \mathrm{L}$. The PCR amplifications were performed using a BioRad thermal cycler (USA). The 
amplified fragments were separated with gel electrophoresis on 1\% agarose. Amplified products, including the mtDNA-complex I gene, were analyzed using a direct sequencing method. The Finch TV software was used for analysis of chromatograms, and bioinformatical analysis was carried out to detect sequence variations (PerkinElmer Company, USA).

\begin{tabular}{ll}
\multicolumn{2}{l}{ Table 1. Mitochondrial primers for PCR-sequencing. } \\
\hline ND1 & ONP82[F] 5'-CTC AAC TTA GTA TTA TAC CC-3' \\
ND2 & ONP84[R] 5'-GAG CTT AGC GCT GTG ATG AG-3' \\
& ONP64[F] 5'-GTC ATC TAC TCT ACC TAC TT-3' \\
ND3 & ONP89[R] 5'-GGC GGG AGA AGT AGA TTG AA-3' \\
& ONP91[F] 5'-CAC TAT CTG CTT CAT CCG CC-3' \\
ND4 & ONP94[R] 5'-GAG CGA TAT ACT AGT ATT CC-3' \\
& ONP9[F] 5'-TCT CCA ACA CAT ATG GCC TA-3' \\
& ONP203[R] 5'-ACT GTG AGT GCG TTC GTT CGT AGT TTG AG-3' \\
ND4L & ONP14[F] 5'-GCG CAG TCA TTC TCA TAA TC-3' \\
ND5 & ONP46[R] 5'-TTT GTT AGG GTT AAC GAG GG-3' \\
& ONP93[F] 5'-TCT GGC CTA TGA GTG ACT AC-3' \\
& ONP203[R] 5'-ACT GTG AGT GCG TTC GTT CGT AGT TTG AG-3' \\
& ONP11[F] 5'-TTT TGG TGC AAC TCC AAA-3' \\
ND6 & ONP74[R] 5'-GGT TGA CCT GTT AGG GTG AG-3' \\
& ONP21[F] 5'-GCA GTC TGC GCC CTA CA-3' \\
& ONP12[R] 5'-TCA GGG TTC ATT CGG GAG GA-3' \\
\hline & ONP204[F] 5'-CTC CAA AGA CCA CAT CAT CGA AAC-3' \\
& ONP318[R] 5'-TTC ATC ATG CGG AGA TGT TGG ATG GGG TGG-3' \\
\hline
\end{tabular}

\section{RESULTS}

To identify genetic causes of complex I activity decrease and investigate the association of complex I mutations with the pathogenesis of MS, we analyzed mtDNA-encoded complex I subunit genes. Several sequence variations in NADH dehydrogenase (ND) subunit, ND2, ND3, ND4, and ND6, were found. One patient had a 4143A $>$ G variation in ND2, and 2 patients had a $10142 \mathrm{C}>\mathrm{T}$ variation in ND3. Eight patients displayed nucleotide changes in ND4, including 1 patient with $11214 \mathrm{C}>\mathrm{T}, 2$ patients with $11343 \mathrm{~T}>\mathrm{C}$, and 3 patients with $11934 \mathrm{~T}>\mathrm{C}$ (Table 2). In 2 patients, a novel variation in the ND4 gene was detected as $12062 \mathrm{C}>\mathrm{T}$. The other variation in ND4 was detected in 11 patients and was located at position $12662 \mathrm{~A}>\mathrm{G}$. Finally, in 2 patients, 2 sequence variations in ND6 were detected: $14179 \mathrm{G}>\mathrm{A}$ and $14263 \mathrm{~T}>\mathrm{C}$. No alterations were found in the ND1, ND4L, and ND5 genes.

Table 2. List of mtDNA sequence variations in the MS patients studied.
\begin{tabular}{llccc}
\hline No. & Nucleotide position & Nucleotide change & $\mathrm{N}(\%)$ \\
\hline 1 & ND1 & - & - & 0 \\
2 & ND2 & 4143 & $\mathrm{~A}>\mathrm{G}$ & 1 \\
3 & ND3 & 10142 & $\mathrm{C}>\mathrm{T}$ & 1 \\
4 & 11214 & $\mathrm{~T}>\mathrm{C}$ & 2 \\
& ND4 & 11343 & $\mathrm{~T}>\mathrm{C}$ & 3 \\
& & 11934 & $\mathrm{C}>\mathrm{T}$ & 2 \\
& & 12062 & $-\mathrm{G}$ & 11 \\
5 & ND4L & - & - & 0 \\
6 & ND5 & - & $\mathrm{G}>\mathrm{A}$ & 1 \\
7 & ND6 & 14179 & $\mathrm{~T}>\mathrm{C}$ & 1 \\
\hline
\end{tabular}




\section{DISCUSSION}

Several studies have confirmed the hypothesis that mitochondria are involved in MS pathogenesis. We know that mtDNA changes play a central role in neurodegenerative diseases, and abnormalities in complex I subunits can lead to neurodegeneration and demyelization (Kumleh et al., 2006). Leber's hereditary optic neuropathy, which is caused by mtDNA mutations, is characterized by symptoms of inflammatory demyelization similar to those in MS (Yu et al., 2008). Additional evidence supports predominantly maternal transmission and a maternally inherited genetic factor in MS (Kumleh et al., 2006). Furthermore, haplotype analysis and restriction site polymorphism have revealed that mtDNA haplogroups $\mathrm{K}$ and $\mathrm{J}$ are associated with MS (Houshmand et al., 2005; Kumleh et al., 2006). Biochemical studies have shown that catalytic activity of complex I is significantly decreased in MS patients compared with that in control subjects (Kumleh et al., 2006). Although some sequence alterations including reported and novel variations were detected in mtDNA-encoded complex I subunit genes in this study, we found no reported pathogenic mutations. The novel nucleotide changes in mtDNA found in this study were screened in healthy normal controls and these may offer new variations related to MS pathogenesis, but functional analysis is necessary to confirm this association.

\section{ACKNOWLEDGMENTS}

We thank the National Institute of Genetic Engineering and Biotechnology for valuable comments and all the participants whose collaboration and permission allowed us to carry out and publish this research.

\section{REFERENCES}

DiMauro S and Schon EA (2001). Mitochondrial DNA mutations in human disease. Am. J. Med. Genet. 106: $18-26$. DiMauro S and Schon EA (2003). Mitochondrial respiratory-chain diseases. N. Engl. J. Med. 348: 2656-2668.

Fauser S, Luberichs J, Besch D and Leo-Kottler B (2002). Sequence analysis of the complete mitochondrial genome in patients with Leber's hereditary optic neuropathy lacking the three most common pathogenic DNA mutations. Biochem. Biophys. Res. Commun. 295: 342-347.

Ghabaee M, Omranisikaroudi M, Amrisaroukolaei S, Meysamie A, et al. (2009). Mitochondrial mutation in Iranian patients with multiple sclerosis, correlation between haplogroups H, A and clinical manifestations. Cell Mol. Neurobiol. 29: 341-346.

Houshmand M, Sanati MH, Babrzadeh F, Ardalan A, et al. (2005). Population screening for association of mitochondrial haplogroups BM, J, K and M with multiple sclerosis: interrelation between haplogroup J and MS in Persian patients. Mult. Scler. 11: 728-730.

Kumleh HH, Riazi GH, Houshmand M, Sanati MH, et al. (2006). Complex I deficiency in Persian multiple sclerosis patients. J. Neurol. Sci. 243: 65-69.

Mao P and Reddy PH (2010). Is multiple sclerosis a mitochondrial disease? Biochim. Biophys. Acta 1802: 66-79.

Smeitink J, van den Heuvel L and DiMauro S (2001). The genetics and pathology of oxidative phosphorylation. Nat. Rev. Genet. 2: 342-352.

Yu X, Koczan D, Sulonen AM, Akkad DA, et al. (2008). mtDNA nt13708A variant increases the risk of multiple sclerosis. PLoS One 3: e1530.

Zeviani M and Di Donato S (2004). Mitochondrial disorders. Brain 127: 2153-2172. 